\title{
Neue Ansätze im integrierten Hochwassermanagement: Floodplain Evaluation Matrix FEM, flussmorphologischer Raumbedarf FMRB und räumlich differenziertes Vegetationsmanagement VeMa
}

\begin{abstract}
Zusammenfassung: In diesem Artikel werden drei neue Ansätze im integrierten Hochwassermanagement vorgestellt, welche in FloodRisk II entwickelt oder optimiert wurden. (I) Die Floodplain Evaluation Matrix (FEM) ermöglicht die objektive Evaluierung von Überflutungsflächen in einem Einzugsgebiet und/oder einer Flussstrecke aus hydrologisch/hydraulischer, ökologischer und soziologischer Sicht. (II) Der flussmorphologische Raumbedarf (FMRB) zeigt sich insbesondere bei

buildings or other higher value usages are allowed. If possible, the potential river morphological space demand, defined by the potential river floodplain system, should be aimed for. (III) The spatially variable vegetation management suggests a differentiation of the river into vegetation dynamic zones, transition zones and sensitive zones with different widths and densities of the vegetation, in order to improve the retention (hydrological) and minimize the water level (hydraulic).
\end{abstract} großen Hochwässern und wurde auf Basis der Untersuchungen minimal mit der insgesamt drei- bis siebenfachen Flussbreite (gemessen ab Böschungsoberkante) festgelegt, wo weder Bauten noch höherwertige Nutzungen zulässig sind. Wo möglich, sollte beim $F M R B$ eine Annäherung an das potenzielle Fluss-Auensystem (maximaler FMRB) erfolgen. (III) Mit dem räumlich differenzierten Vegetationsmanagement $\left(\mathrm{VeMa}_{\mathrm{Flood}}\right)$ erfolgt eine Einteilung der Flussabschnitte in vegetationsdynamische Strecken, Übergangsstrecken und sensible Strecken mit unterschiedlicher Breite und Dichte der Vegetation, um damit eine verstärkende Retentionswirkung (hydrologisch) oder Minimierung des Wasserspiegels (hydraulisch) zu ermöglichen.

New approaches to integrated flood management: Floodplain Evaluation Matrix (FEM), minimum river morphological space demand (FMRB), and spatially variable vegetation management $\left(\mathrm{VeMa}_{\mathrm{FLOOD}}\right)$

Summary: This paper presents three new approaches within the integrated flood management, which have been developed or optimized in FloodRisk II. (I) The Floodplain Evaluation Matrix (FEM) allows an objective evaluation of inundation areas within a river basin and/or river reach from hydrological/hydraulic, ecological and sociological viewpoint. (II) The minimum river morphological space demand is to be seen especially after large floods and was defined, based on the analysis of the recent extreme floods, as three to sevenfold the existing river bed width (measured from the river banks), where no

\section{Einleitung}

Die Forderung „Flüsse brauchen Raum“ ist nicht neu, wurde sie bereits seit den extremen Hochwässern 1965/66 aufgestellt. In der Praxis zeigte sich demgegenüber ein weiter fortschreitender Flächenverbrauch. Der tägliche Verbrauch für Siedlungs- und Verkehrstätigkeit liegt bei 12 Hektar und der Gesamtflächenverbrauch (inkl. Sportflächen, Abbauflächen, usw.) liegt bei knapp 22 Hektar (Durchschnitt der 3 Jahres-Periode 2006-2009; Umweltbundesamt, 2009). Dabei sind insbesondere Überflutungsflächen betroffen, da dort gute Erschließungsmöglichkeiten, Verkehrsanbindung und günstige Preise vorherrschen. Darüber hinaus wurden die Flüsse sehr stark eingeengt, und sie besitzen nicht mehr den flussmorphologisch notwendigen Raum für Veränderungen, die bei großen Hochwässern auftreten. Dies steht zudem in Wechselwirkung mit der Vegetation entlang der Fließgewässer und damit auch mit der Ökologie.

Die natürlichen Überflutungsflächen entlang von Fließgewässern haben signifikanten Einfluss auf Scheitelabfluss und Laufzeit von Hochwasserwellen und spielen zudem eine bedeutende ökologische Rolle. Der Erhalt bestehender bzw. die Wiederherstellung bereits verloren gegangener Überflutungsräume ist somit ein wichtiger Bestandteil eines integrierten Hochwasserrisiko-Managements, wie dies auch in der EU-Richtlinie über die Bewertung und das Management von Hochwasserrisiken (2007 60 EG) gefordert wird.
Sowohl das Hochwasser im Sommer 2002 als auch jenes des Jahres 2005 zeigten die Bedeutung der Geomorphologie für den Ablauf und die Auswirkungen von Extremereignissen. Hierbei veränderte sich die Flussmorphologie und -geometrie teils so stark, dass beispielsweise Breitenzunahmen im Mittel auf das vier- bis fünffache (an der Trisanna bis zum 20fachen) auftraten (Habersack et al., 2009b). Das Ausmaß der morphologischen Veränderungen ist einerseits von den während des Ereignisses ablaufenden Prozessen und andererseits von den langfristigen Entwicklungen wie z. B. Sohleintiefungen abhängig. Übergeordnet ist der Feststoffhaushalt in Einzugsgebieten von großer Bedeutung für die Entwicklung bei Extremereignissen.

Das Management der Vegetation entlang von Flüssen ist gerade bei großen Hochwässern Anlass für Diskussionen, die eine objektive Zugangsweise benötigen.

Allen drei Bereichen ist ein Raumbedarf gemeinsam, der mittels verschiedener Methoden, die im Rahmen von FloodRisk II entwickelt oder optimiert wurden, abgeschätzt und bewertet werden soll. Ziel dieses Artikels ist die Vorstellung der erarbeiteten Methoden, wobei die Bedeutung des Raums sowohl für das integrierte Hochwassermanagement als auch für den ökologischen Zustand dargestellt wird.

\section{Floodplain Evaluation Matrix FEM}

Um die Bemühungen für einen Erhalt bzw. eine Wiederherstellung von Überflutungsräumen unter objektiven Kriterien durchführen zu können, wurde im Rahmen des Era-Net CRUE Forschungsprojektes „Flood risk reduction by PReserving and restOring river Floodplains - PRO_Floodplain" eine Methode zur Evaluierung von Überflutungsräumen entwickelt, die hydromorphologischen, ökologischen und soziologischen Gesichtspunkten Rechnung trägt (Habersack et al., 2008a). Diese „Floodplain Evaluation Matrix“ FEM ermöglicht es, verschiedene Überflutungsräume aber auch verschiedene Szenarien hinsichtlich dieser drei Themen miteinander zu verglei- 


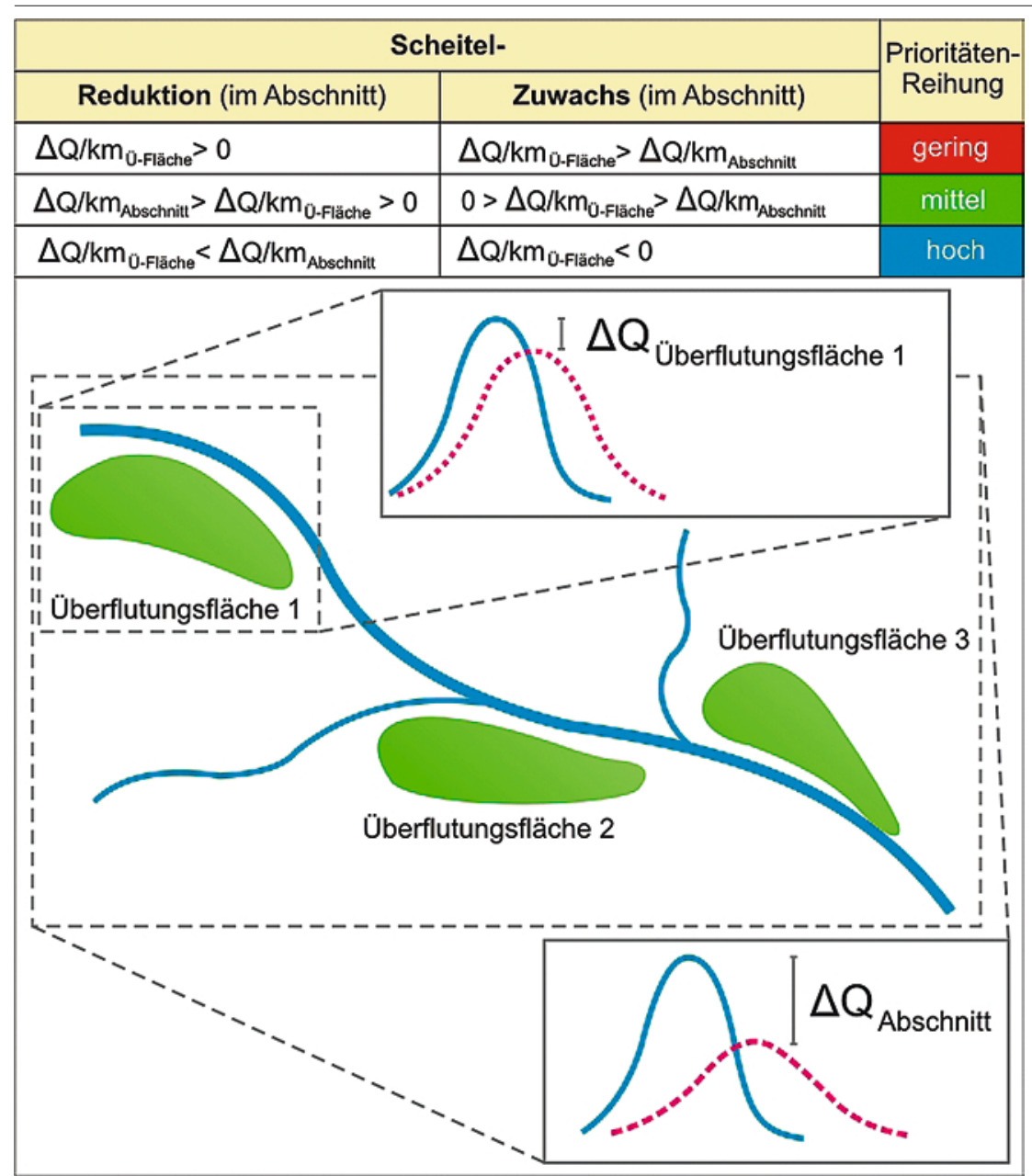

Abb. 1: FEM-Beurteilung für den Parameter Scheitelreduktion $\triangle Q$ (Habersack et al., 2008a).

chen und eine Bewertung und Prioritätenreihung innerhalb eines Flusseinzugsgebietes oder einer Flussstrecke vorzunehmen.

\subsection{Hydromorphologie (Hydrologie / Hydraulik)}

Die hydromorphologische Evaluierung beurteilt vor allem das Retentionsverhalten der Überflutungsflächen und die hydraulische Wirkung im Hochwasserfall unter den gegebenen geomorphologischen Bedingungen und strebt einen Vergleich verschiedener Szenarien in Hinblick auf die Effekte bei Hochwasser an. Hierbei stellt die hydrologische Evaluierung eine einzugsgebietsbezogene Betrachtung dar (Wirkung auf Unterlieger), während sich die hydraulische Evaluierung auf lokale Effekte bezieht (naheliegende Siedlungen).

\section{FEM-Parameter Hydrologie}

- Scheitelreduktion: Die auf Flusskilometer bezogene, standardisierte Scheitelreduktion des Flussabschnittes $(\Delta \mathrm{Q}$ / $\left.\mathrm{km}_{\text {Abschnit }}\right)$ wird mit jenen der einzelnen Überflutungsflächen $\left(\Delta \mathrm{Q} / \mathrm{km}_{\text {Überllutungs- }}\right.$ flïche) verglichen (siehe $A b b .1$ ).

- Scheitelverzögerung: Vergleich von $\Delta \mathrm{t} /$ $\mathrm{km}_{\text {Abschnitt }}$ mit $\Delta \mathrm{t} / \mathrm{km}_{\text {Überflutungsfläche }}$.

- Flusskilometer bezogene Kenngrößen: Beurteilung von aktuellem und potenziellem Retentionsvolumen, Vorlandbreite und -rauigkeit etc.

- Sekundäre Effekte für höhere Abflüsse: Wirkung bei Abflüssen größer als der Bemessungsabfluss (Restrisiko [z. B. Dammbruch], erhöhtes Risiko [ $\left.\left.\mathrm{HQ}_{300}\right]\right)$.

- Andere Prozesse: qualitative Aussagen zu Sedimenttransport und Totholz in Abhängigkeit zu ihrem räumlich differenzierten Auftreten.

\section{FEM-Parameter Hydraulik}

- Wasserspiegelhöhen: je nach Lage im Gesamtsystem (Auswirkungen auf besiedeltes Gebiet) werden Wasserspiegellagen-Änderungen positiv oder negativ gewertet.

- Fließgeschwindigkeiten: räumlich differenzierte Beurteilung der Veränderun- gen in den Fließgeschwindigkeiten.

- Sohlschubspannungen: Die Veränderungen der Sohlschubspannungen werden auf erwünschte bzw. unerwünschte Folgen untersucht.

- Spezifischer Durchfluss: Beurteilung der lokalen Bedeutung des Vorlanddurchflusses.

\section{2. Ökologie}

Die ökologischen Untersuchungen evaluieren die Bedeutung von Überflutungsflächen für die Biozönosen in und am $\mathrm{Ge}$ wässer und stellen somit eine wichtige Grundlage in Hinblick auf die Forderungen der EU-Wasserrahmenrichtlinie dar.

\section{FEM-Parameter Ökologie}

- Angepasste Landnutzung: Beurteilung der Überflutungsverträglichkeit.

- Dynamik des Wasserspiegels: Evaluierung der Natürlichkeit der Wasserspiegelschwankungen.

- Fließgeschwindigkeiten: Bewertung, ob diese mit den Biozönosen verträglich sind.

- Gelände-Relief: Bewertung der strukturellen Vielfalt.

- Konnektivität der Wasserkörper: in longitudinaler, lateraler und vertikaler Hinsicht.

- Potenzial/Gefährdung für die Entwicklung typischer Lebensräume: gemäß der EU-FFH-Richtlinie.

\subsection{Soziologie}

Die soziologische Evaluierung untersucht jene Rahmenbedingungen, die bei der Durchführung von Projekten im Sinne eines Erhalts bzw. einer Wiederherstellung von Überflutungsflächen aus gesellschaftspolitischer Sicht zu beachten sind.

\section{FEM-Parameter Soziologie}

- Art der Nutzung: Anzahl der verschiedenen GrundeigentümerInnen sowie deren Organisation in Interessensvertretungen etc.

- Kommunikationskanäle: lokale Repräsentation und Rolle in den Kommunikationskanälen der maßgebenden AkteurInnen.

\subsection{Interdisziplinäres Ergebnis:} Floodplain Evaluation Matrix FEM

Nach der sektoralen Evaluierung in den drei Bereichen Hydromorphologie, Ökologie und Soziologie werden die Ergebnisse in der FEM zusammengefasst. Die 


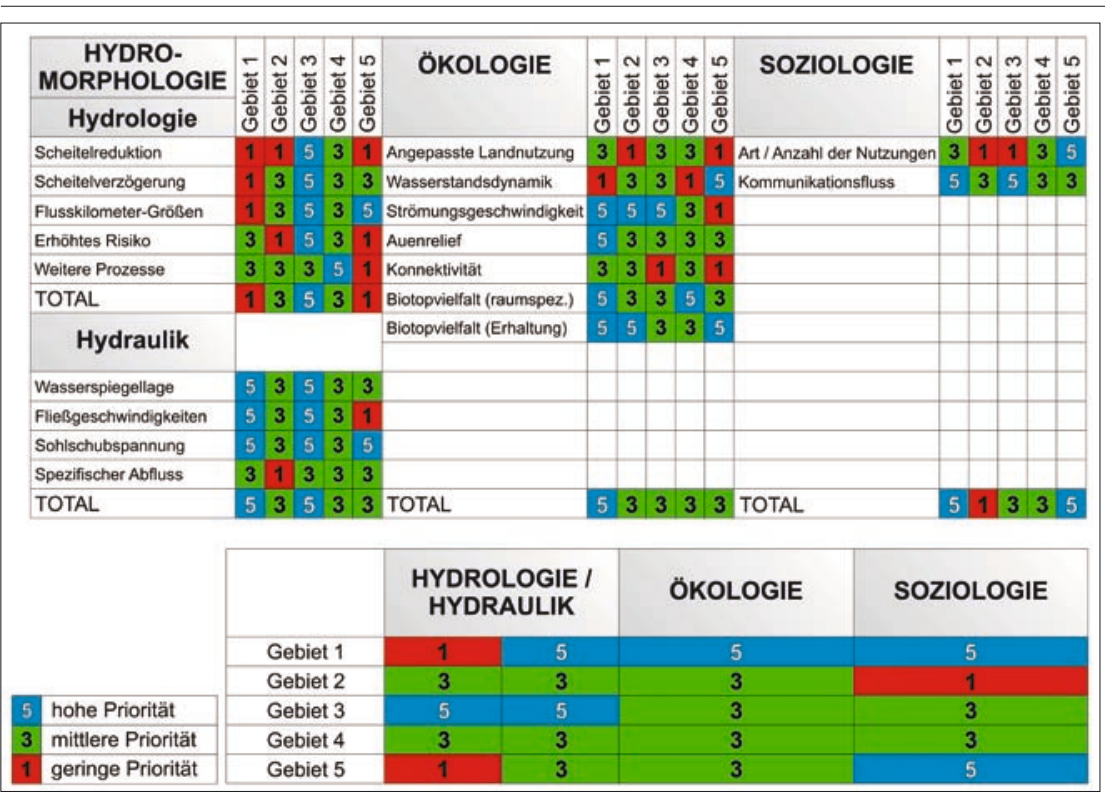

Abb. 2: Beispiel für eine vergleichende Evaluierung von fünf verschiedenen Gebieten mit Überflutungsflächen innerhalb der FEM-Matrix (Habersack et al., 2008a).

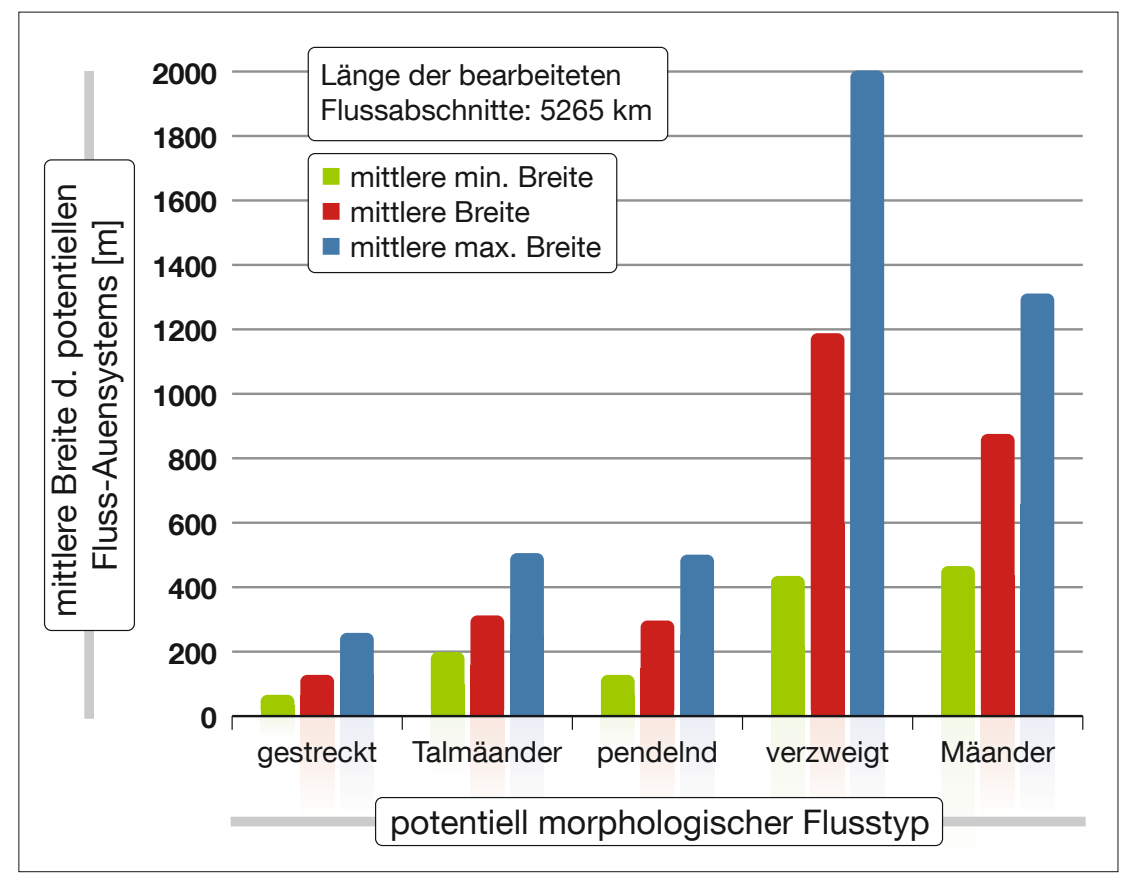

Abb. 3: Mittlere Breiten des potenziellen Fluss-Auensystems, differenziert nach morphologischen Flusstypen (Muhar et al., 2009).

Evaluierung der einzelnen FEM-Parameter führt zu einer Gesamtevaluierung in jedem Bereich und in weiterer Folge zu einer Klassifizierung und Prioritätenreihung (siehe Abb. 2).

Die FEM Prioritätenreihung ergibt, wo die Erhaltung und Verbesserung von Überflutungsflächen am besten wirkt und der Fokus auf Schutz- und Wiederherstellungsmaßnahmen gelegt werden soll. Die FEM-Methode ergibt nicht, dass Überflutungsflächen mit geringer Priorität keinen
Wert haben oder nicht für das Hochwasserrisikomanagement erforderlich sind. Derartige Überflutungsflächen besitzen insbesondere für das erhöhte Risiko eine hohe Bedeutung (auch in Hinblick auf den Klimawandel).

Die Interdisziplinäre Floodplain Evaluation Matrix (FEM) liefert folgende Ergebnisse:

- Integrale Planung von technischen, ökologischen und soziologischen Aspekten.
- Methode zur Evaluierung von Überflutungsflächen, welche erhalten oder wiederhergestellt werden sollten.

- Geeignetes Werkzeug für EntscheidungsträgerInnen zur Verringerung des Hochwasser-Risikos durch nichtstrukturelle Maßnahmen.

- Die FEM-Ergebnisse können als gesetzliche Einschränkungen in Bezug auf die Verwendung von Überflutungsflächen angewendet werden, wenn diese für ein integrales Hochwasser-Risiko-Management relevant sein sollten.

- Nachhaltige Nutzung der Wasserkörper durch Unterstützung der natürlichen Funktionen.

- Verbesserung der Beziehung zwischen Gesellschaft und Umwelt.

\section{Flussmorphologischer} Raumbedarf FMRB

Im Zuge der Hochwasserereignisse der Jahre 2002 und 2005 zeigte sich, dass bei extremen Hochwasserereignissen nennenswerte Veränderungen der Flussmorphologie auftreten und dadurch gravierende Konsequenzen für die Abflusssituation entstehen.

Das Hochwasser im August 2002 in Niederösterreich am Kamp, aber auch in Oberösterreich z. B. an der Aist ergab, dass durch signifikante Sohlbreitenänderungen während des Ereignisses völlig andere hydraulische Gegebenheiten geschaffen wurden (Hauer \& Habersack, 2009). Dies führte einerseits zu Entlastungen, da durch den höheren Abflussquerschnitt ein Absinken des Hochwasserspiegels auftrat, andererseits wurden infolge der Verwerfungen in Siedlungsgebieten und bei Infrastruktureinrichtungen deutlich höhere Schäden verursacht (Habersack et al., 2004). Das Hochwasser im August 2005 trat schwerpunktartig im alpinen Raum auf. Aufgrund der Beengtheit der Tallagen und des hohen Nutzungsdruckes war das Schadensbild infolge Verwerfungen deutlich höher.

Im Flussraum sind unterschiedliche Flächenansprüche vorhanden. Siedlungsräume sind mit schutzwasserbaulichen Maßnahmen vor Überflutung bei einem $\mathrm{HQ}_{100}$ zu schützen.

Als potenzielles Fluss-Auensystem ist jener Talraum definiert, der unter natürlichen Verhältnissen durch das Fließgewässer geprägt wird bzw. wurde und gibt damit die maximale laterale Ausdehnung und den Raumbedarf eines Fließgewässers unter natürlichen Bedingungen an (Muhar et al., 2003). Dieser maximale 
flussmorphologische Raumbedarf vor den Zeiten der systematischen Flussregulierung, wie auf historischen Karten ersichtlich, erstreckte sich oft auf den gesamten Talboden. Durch Regulierungsmaßnahmen wurde der Raum für Flüsse in Österreich stark eingeschränkt (Muhar et al., 2009). Die Ergebnisse der Analyse in FloodRisk II über das Ausmaß der Längsverbauung zeigen an $68 \%$ der bearbeiteten Gewässerabschnitte (3.550 Fkm) der großen Flüsse Österreichs mit einem Einzugsgebiet über $500 \mathrm{~km}^{2}$ eine durchgehende bzw. beinahe durchgehende Längsverbauung (Muhar et al., 2009). Bereichsweise Längssicherungen weisen $21 \%$ (1.093 Fkm) der Fließgewässerabschnitte auf. Keine oder nur lokale Sicherungsmaßnahmen sind nur an $11 \%$ (555 Fkm) der Flussstrecken vorhanden.

Abbildung 3 gibt einen Überblick über die mittleren Breitenverhältnisse im potenziellen Fluss-Auensystem von 53 bearbeiteten Fließgewässern mit einem Einzugsgebiet über $500 \mathrm{~km}^{2}$ in Abhängigkeit vom potenziellen morphologischen Flusstyp (Muhar et al., 2009). Die geringsten durchschnittlichen Breitenausdehnungen finden sich bei gestrecktem und pendelndem Flusstyp sowie beim Talmäandertyp. Mäandrierende Flussabschnitte erreichen mittlere Breiten von über $800 \mathrm{~m}$, verzweigte Furkationssysteme sogar eine mittlere maximale Breite von $2 \mathrm{~km}$.

Gegenüber dem potenziellen FlussAuensystem besteht in intensiv genutzten Talräumen eine sehr reduzierte aktuelle (meist regulierte) Breite. Stark anthropogen veränderter Flussverlauf korreliert mit einer Zunahme der intensiven Umlandnutzung (Ackerflächen, Siedlung) des Talbodens. Abschnitte mit bogigem oder linearem Flussverlauf sind durch intensives Grünland oder Acker geprägt. Die Ergebnisse der Landnutzungsanalysen sind in Hinblick auf den Hochwasserschutz interessant. Über die Verteilung der Landnutzungstypen ist - auf genereller Ebene - das Rückhaltevermögen des Talraums („Hochwasserpräventionsfunktion“) bzw. eine mögliche Abflussverstärkung durch spezifische landwirtschaftliche Kulturen (intensiv bewirtschaftete Ackerflächen v.a. bei Reihenkulturanbau wie bei Mais, Kartoffeln, Rüben, Feldgemüse, Weinbau, Sonnenblumen u.a.) anzusprechen. Gleichzeitig sind anhand der Daten Kulturflächen mit hohem Schadenspotential (siehe dazu Wagner et al., 2009) zu identifizieren.

Die Berechnung des hydrologisch-hydraulischen Abflussraumes $\left(\mathrm{HQ}_{30}, \mathrm{HQ}_{100}\right.$,
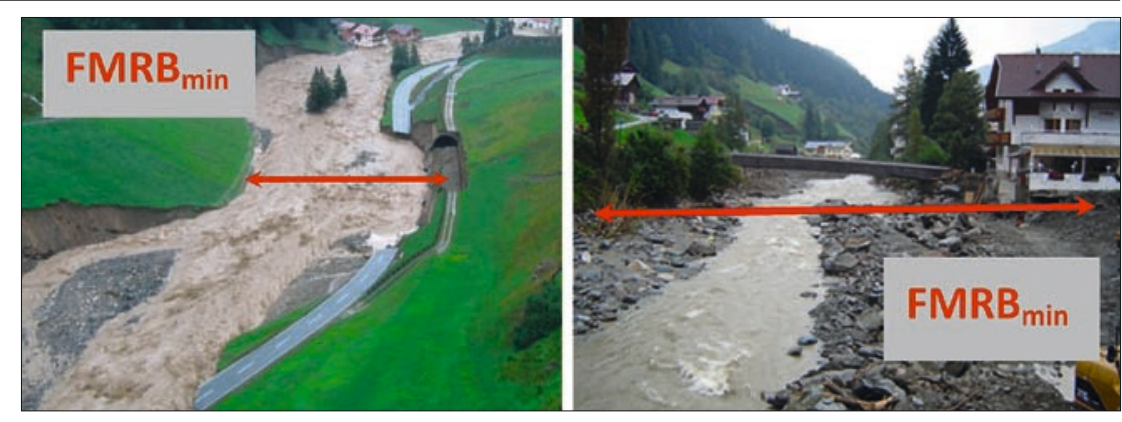

Abb. 4: Darstellung des benötigten minimalen flussmorphologischen Raumbedarfs (FMRBmin) beim Hochwasser 2005 an der Trisanna. (Fotos: ASI, BOKU-IWHW).

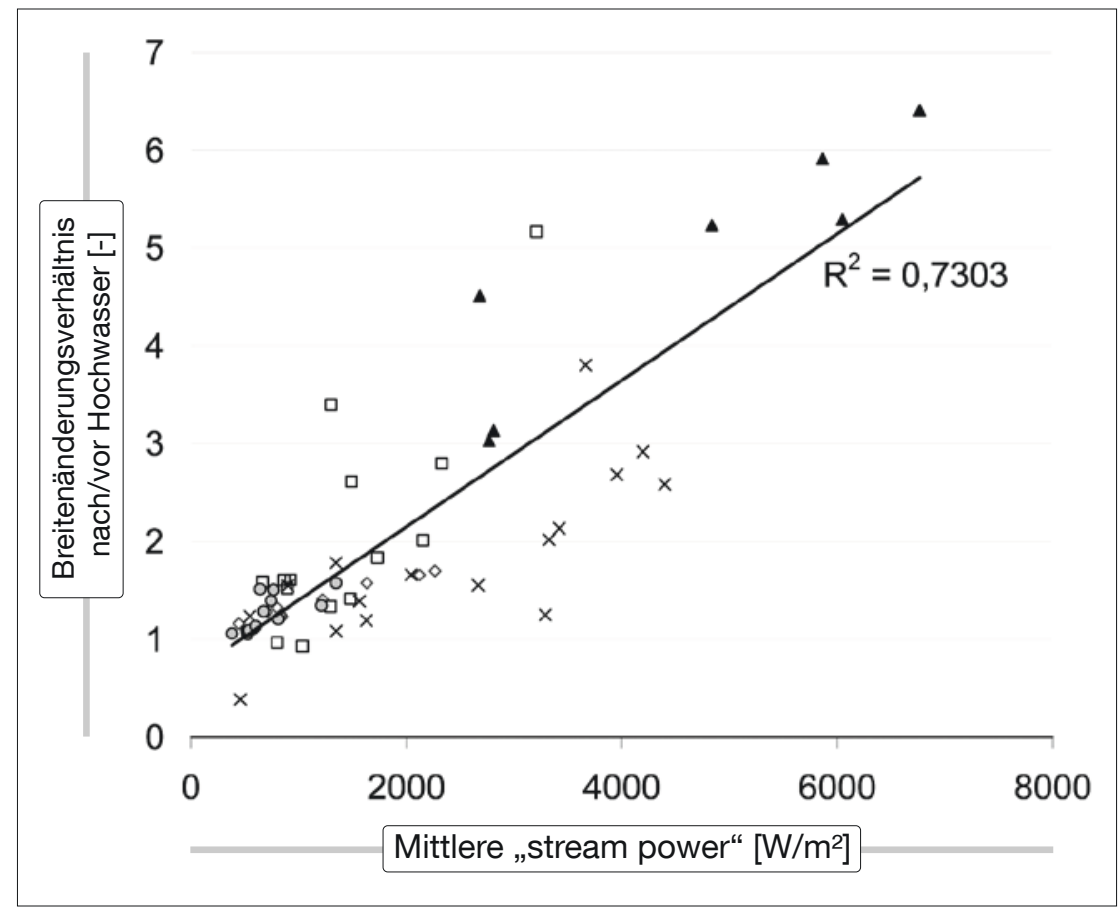

Abb. 5: Zusammenhang der mittleren stream power mit dem mittleren Breitenänderungsverhältnis an 5 alpinen Flüssen (Krapesch et al., submitted).

$\mathrm{HQ}_{300}$ ) wird bereits standardisiert für die Gefahrenzonenplanung verlangt. Diese gehen aber von einer stabilen Gewässermorphologie während des Ereignisses aus.

Als wesentlicher Raumanspruch im Rahmen des Hochwassermanagements ist der minimale flussmorphologische Raumbedarf $\left(\mathrm{FMRB}_{\min }\right)$ zu betrachten. Er umfasst Bereiche im Vorland der Flüsse, die im Hochwasserfall durch morphologische Veränderungen (Verwerfungen, Umlagerungen, Erosionen) gefährdet sind. Der minimale flussmorphologische Raumbedarf definiert sich als minimaler Sicherheitsabstand, um Gebäude, Infrastruktur und sonstige anthropogene Nutzungen vor den erosiven Kräften des Flusses im Hochwasserfall zu schützen (Abb. 4).

Es sollte das Bewusstsein geschaffen werden, dass neben den von Überflutung gefährdeten Flächen die Nahbereiche des Flusses aufgrund unterschiedlicher Einflüsse morphologischen Veränderungen im Hochwasserfall unterliegen können. In Abhängigkeit vom Flusstyp, den Einzugsgebietscharakteristika (z. B. Feststoffhaushalt, Gefälle, Geologie etc.) und den hydrologischen Eigenschaften sowie dem Verbauungszustand und -grad können sich unterschiedliche Flussverbreiterungen während eines Hochwassers ergeben. Mittels Vergleich von hydraulischen Kenngrößen mit dem Breitenänderungsverhältnis nach dem Hochwasser konnte die mittlere spezifische „stream power" als eine von mehreren Kenngrößen zur Ermittlung des $\mathrm{FMRB}_{\text {min }}$ identifiziert werden (Abb. 5, Krapesch et al., in prep). Einzelne Parameter sind jedoch nicht ausreichend, um Breitenänderungen abzuschätzen. Einflüsse unterschiedlicher Art (im Speziellen 


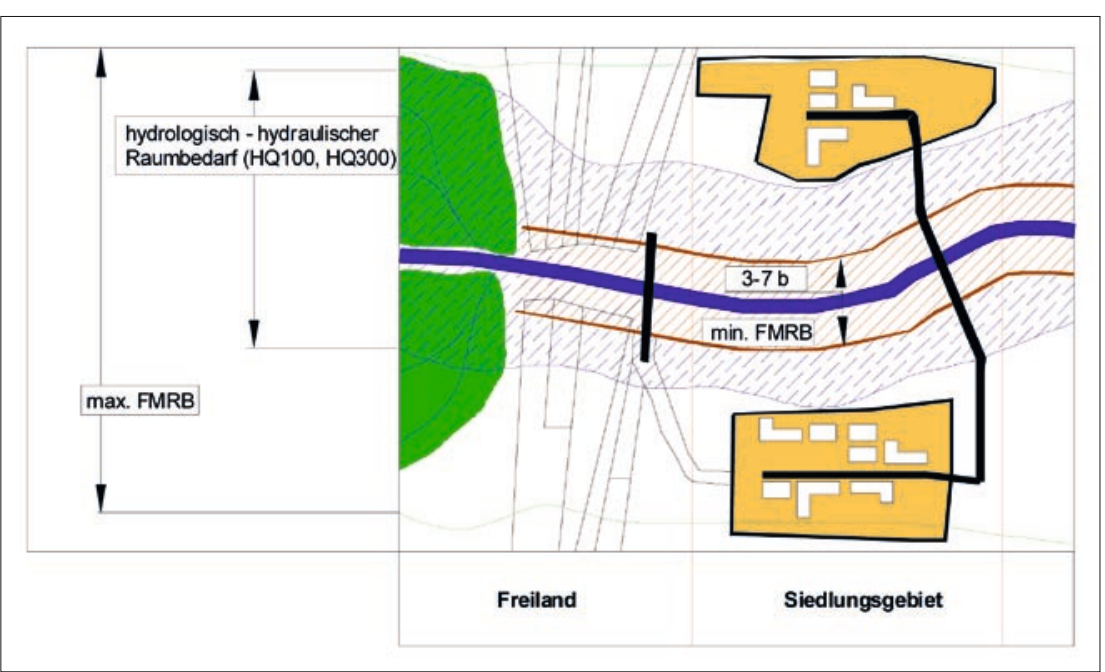

Abb. 6: Schematische Darstellung der Raumansprüche bzw. des flussmorphologischen Raumbedarfs FMRB (Habersack et al., 2009a).

der Geschiebehaushalt und der Widerstand durch die Verbauung etc.) beeinflussen die Werte und reduzieren die Güte der Zusammenhänge.

An der Trisanna konnte ein mittleres Breitenänderungsverhältnis von 3,83 festgestellt werden. Durch die Analyse der historischen Flussbreiten aus der 3. Landesaufnahme von 1864-1887 konnte deutlich gemacht werden, dass diese auch um den Faktor 2,43 größer als die Flussbreite vor dem Hochwasser waren. Es zeigte sich, dass im Bereich von Brücken zahlreiche morphologische Veränderungen stattfanden, und diese daher einer genauen Be- trachtung in Bezug auf mögliche Verwerfungen unterzogen werden müssen (vgl. Hauer und Habersack, 2009). Weiters konnte bei Durchflüssen von $\mathrm{HQ}_{100}$ und größer statistisch eine Häufung von morphologischen Veränderungen festgestellt werden.

\subsection{Langfristige Entwicklung der Flussmorphologie in Verbindung mit Hochwasser}

Langfristige Sedimentdefizite in Verbindung mit verringerter Gerinnebreite, unterbundener Seitenerosion und erhöhtem
Sohlgefälle ergeben Handlungsbedarf, um den Hochwasserschutz nicht zu gefährden und den guten ökologischen Zustand zu erreichen. Sedimentdefizite durch Kontinuumsunterbrechungen und Geschiebebewirtschaftung sowie erhöhte Transportkapazität infolge Regulierungen fördern die Sohleintiefung. Ein langfristiges Ungleichgewicht im Geschiebehaushalt (Geschiebedefizit oder -überschuss) führt daher zu morphologischen Veränderungen der Gewässersohle, die in der Regel langsam ablaufen. Durch diese Veränderungen können zwar langsam, aber damit unter Umständen unbemerkt Situationen entstehen, die bei extremen Hochwasserereignissen zu Gefährdungen führen.

\subsection{Empfehlungen}

Erfüllung des minimalen flussmorphologischen Raumbedarfes $\left(\mathrm{FMRB}_{\text {min }}\right)$ : Erhaltung/Herstellung eines minimalen Sicherheitsabstandes der ein- bis dreifachen Flussbreite (aktuelle Breite zwischen Böschungsoberkanten) links- und rechtsufrig mit absolutem Bebauungsverbot. Insgesamt sollte somit zumindest die 3 bis 7 fache Flussbreite freigehalten werden, um Flächen für die morphologischen Veränderungen bei großen Hochwasserereignissen zur Verfügung zu haben und damit Schäden zu verringern ( $A b b$. 6). Wichtig ist der Schutz und die Erhaltung der vorhandenen gewässertypspezifischen Flussabschnitte, die Erhaltung und Vergrößerung

\section{LITERATUR}

Florineth F (2004) Pflanzen statt Beton. Handbuch zur Ingenieurbiologie und Vegetationstechnik. Berlin - Hannover. Pratzer Verlag.

Habersack H, Bürgel J, Petraschek A (2004) Analyse der Hochwasserereignisse vom August 2002 - FloodRisk. Wien, Bundesministerium für Land- und Forstwirtschaft, Umwelt und WasserLand- und Forstwits

Habersack H, Hauer C, Schober B, Dister E, Quick I, Harms O, Wintz M, Piquette E, Schwarz U (2008a) Flood risk reduction by PReserving and restOring river FLOODPLAINs PRO FLOODPLAIN, European Conference on Flood Risk Management Research into Practice, Oxford, England (FloodRISK 2008, ed. P. Samuals, S. Huntington, W. Allsop, J. Harrop), CRC Press Taylor \& Francis Group, pp. 733-743.

Habersack H, Hofbauer S, Hauer C

(2008b) Vegetation impacts on flood flows evaluation of flow resistance based on a hydraulic scale model and numerical hydrodynamic modelling, In: Altinakar, M.S., Kokpinar, M.A. Darama, Y., Yegen, E.B., Harmancioglu, N. (Eds.), International Conference on Fluvial Hydraulics, River Flow 2008, 1, 425-433; ISBN: 978-605-60136-1-4

Habersack H, Bürgel J, Kanonier A (2009a) FloodRisk II, Vertiefung und Vernetzung zukunftsweisender Umsetzungsstrategien zum integrierten Hochwassermanagement, Wien, Bundesministerium für Land- und Forstwirtschaft,
Umwelt und Wasserwirtschaft: 1-259 Habersack H, Krapesch G, Jäger E, Hauer C, Hengl M, Breitenbaumer G, Kreinz N, Wagne B (2009b) Darstellung der Auswirkungen von Änderungen im Feststoffhaushalt und der Flussmorphologie auf den Hochwasserabfluss TP2.1 morphologie

Hauer C, Habersack H (2009) Morphodynamics of a 1000-year flood in the Kamp River, Austria, and impacts on floodplain morphology. Earth Surf Process Landf.; 34(5): 654-682.

Habersack H, Jäger E, Wagner B, Hauer C, Preis S (2009c) Vegetation und Hochwasser aus hydraulischer und ökologischer Sicht, TP 4.2 hydraulischer

FloodRisk II.
Hochwasserrichtlinie, Richtlinie 2007/60/EG des Europäischen Parlaments und des Rates vom 23.Oktober 2007 über die Bewertung und das Management von Hochwasserrisiken.

Hofbauer S, Preis S, Muhar S, Hauer C, Jungwirth M, Habersack H (2006) Management der Vegetation in einer Flusslandschaft - innovative Herangehensweise aus hydraulischer und ökologischer Sicht am Beispiel Kamp. Österreichische Wasser- und Abfallwirtschaft 58 (11-12): 185-192.

Jungwirth M, Haidvogel G, Moog O, Muhar S., Schmutz S (2003) Angewandte Fischökologie an Fließgewässern. Facultas, UTB, Wien. Krapesch G, Hauer C, Habersack H (submitted) Hydromorphological parameters testing on various scales for determining the spatial de- mand of morphological processes in alpine regions of Austria during extreme floods.

Muhar S, Poppe M, Schmutz S, Egger G, Melcher A (2003) Analyse und Ausweisung naturräumlicher Flusstypen Österreichs. Österreichische Wasser- und Abfallwirtschaft, 7-8,

sche Wass

Muhar S, Poppe M, Weiss M, Preis S, Pohl G, Hohensinner S, Hein T (2009) Ökologie und Hochwasserschutz (EU-National) Auswirkungen der WRRL. TP4.3 FloodRisk II.

Schmutz S, Melcher A, Muhar S, Zitek A,

Poppe M, Trautwein C, Jungwirth M

(2007) MIRR - Model-based Instrument for River Restoration. Entwicklung eines strategischen Instruments zur integrativen Bewertung ökologischer Restaurationsmaßnahmen an Fließgewässern. Studie im Auftrag von Lebensministerium und Land Niederösterreich, Wien.

Schwarz U, Lazowski W, Exner A, Angermann

K, Egger G, Götzl M, EssI F, Peterseil J (2009) Aueninventar Österreichs. Modul 2: Bearbeitung der Bundesländer Steiermark und Kärnten und Basisbearbeitung Gesamtösterreich. TP4.4 FloodRisk II. Wien, $123 \mathrm{~S}$.

Umweltbundesamt (2009) Regionalinformation der Grundstücksdatenbank (Bundesamt für Eich- und Vermessungswesen) aufbereitet durch Umweltbundesamt (www.umweltbundesamt.at). Wagner K, Janetschek H, Neuwirth J (2009) Landwirtschaft und Hochwasser. TP9.5 FloodRisk II. Bundesanstalt für Agrarwirtschaft, Wien. 
des Fluss-Auenraumes entsprechend der lateralen Ausdehnung und der Morphodynamik des Flusstyps: In Talräumen ohne Besiedlung oder ohne höherwertige Nutzungen sollte aus gewässerökologischer Sicht eine Vergrößerung des FlussAuenraumes in Richtung der flusstypspezifischen Raumausdehnung bzw. des maximalen flussmorphologischen Raumbedarfs angestrebt werden. In Bereichen mit höherwertigen Nutzungen ist auf jeden Fall zumindest der minimale flussmorphologische Raumanspruch (siehe oben) gefordert.

Die Durchführung folgender Restaurationsmaßnahmen an degradierten Flussabschnitten unter Betrachtung des gesamten potenziellen Fluss-Auensystems ist aus ökologischer Sicht anzustreben:

Initiieren/Entwickeln des morphologischen Flusstyps, Restauration des potenziellen Fluss-Auensystems inklusive der Vergrößerung/Wiederanbindung von Überflutungsräumen bzw. Initiieren/Anlage von auentypischen Habitaten (Schmutz et al., 2007) und Extensivierung der Umlandnutzung in der $\mathrm{HQ}_{30}$-Zone. Dabei könnten die in TP 4.4. Aueninventar (Schwarz et al., 2009) ausgewiesenen Auengebiete hot spots für flussdynamische Entwicklungen - im Sinne einer großflächigen Restauration des Fluss-Auensystems - darstellen.

Weiters sollte das Feststoffpotenzial in Abhängigkeit von den Eigenschaften des Einzugsgebietes bei zukünftigen Hochwasserschutzplanungen stärker berücksichtigt werden und die Erhaltung und Wiederherstellung des Sedimentkontinuums und der flusstypspezifischen Morphodynamik erreicht werden.

\section{Räumlich differenziertes Vegetationsmanagement $\mathrm{VeMa}_{\text {FLOOD }}$}

Die Vegetation hat im Hochwasserfall einen enormen Einfluss auf das Abflussverhalten eines Flusses (Habersack et al., 2009c). Der Bewuchs im Vorland bewirkt eine Reduktion der Fließgeschwindigkeit und damit verbunden einen Anstieg des Wasserspiegels. Breite und Dichte der Vegetation stellen hierbei die entscheidenden Parameter dar. Je breiter und dichter die Vegetation im Vorland bzw. Uferbereich ausgeprägt ist, desto größer ist die zu erwartende Aufspiegelung (Habersack et al., 2008b). Zudem schützt die Vegetation den Boden vor Erosionsschäden und begünstigt andererseits die Sedimentation von Schwebstoffen.

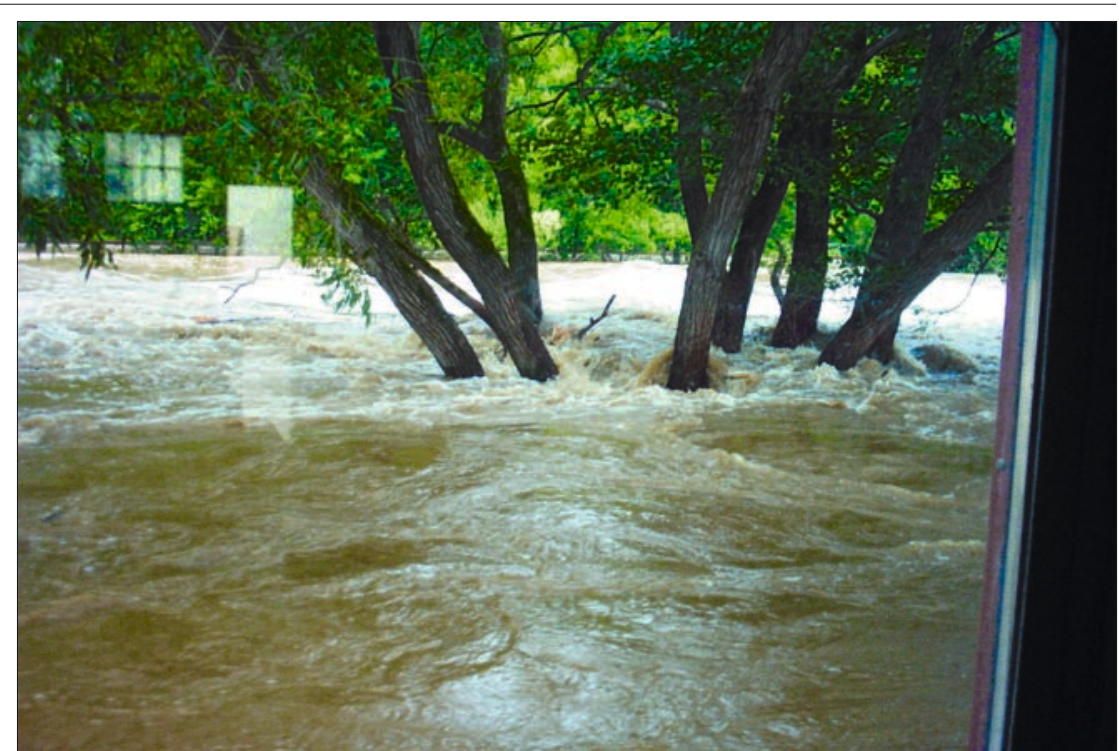

Abb. 7: Einfluss von Vegetation auf das Hochwasserabflussgeschehen: Pflanzen stellen Rauigkeitselemente dar, d.h. sie reduzieren lokal die Fließgeschwindigkeit und damit die Sohlschubspannung des Wassers (Habersack et al., 2009b).

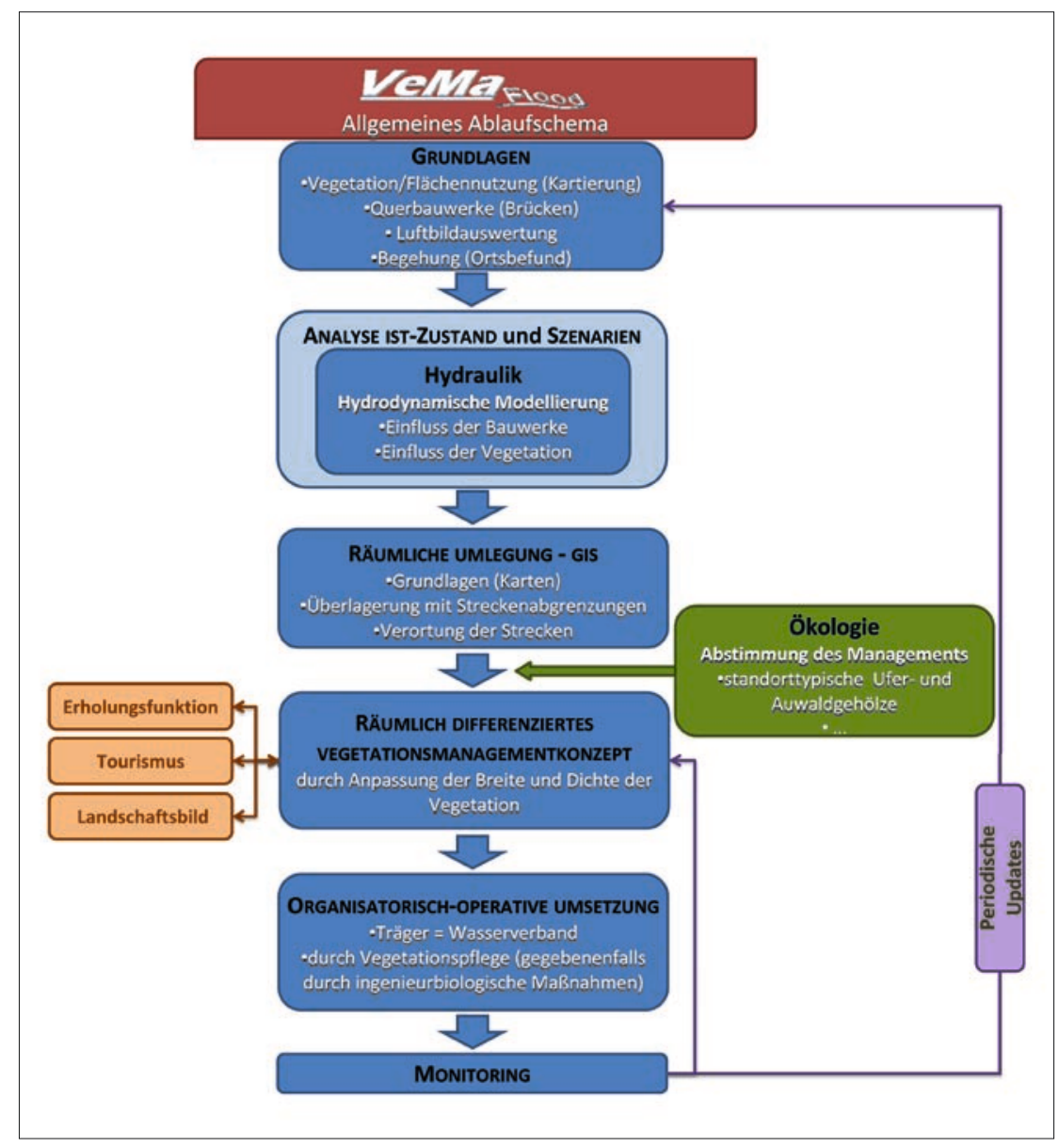

Abb. 8: Allgemeines Ablaufschema des räumlich differenzierten Vegetationsmanagementkonzepts $\left(\mathrm{VeMa}_{\mathrm{FLOOD}}\right)$ (Habersack et al., 2009c).

Für die Verklausungsgefahr von Brücken im Fall eines Hochwassers sind der Abstand zwischen der Wasseroberfläche und der Konstruktionsunterkante (lichte
Weite) und das Vorhandensein von Pfeilern ausschlaggebend (Habersack et al., 2004). Deshalb sollte beim Neubau von Brücken eine pfeilerlose Bauweise, glatte 
abweisende Verschalungen der Brückenunterseite sowie große Durchflussöffnungen (lichte Breite) angestrebt werden.

Entlang eines Fließgewässers ist die Bedeutsamkeit von Vegetation und Totholz hinsichtlich ökologischer Aspekte unumstritten und zeigt sich vor allem durch eine erhöhte Strukturvielfalt sowohl im Gewässer selbst als auch im Uferbereich, durch Schaffung von Habitaten, Refugien und Brutmöglichkeiten sowie durch eine wichtige Nahrungsquelle (Jungwirth et al., 2003, Florineth, 2004).

\subsection{Vegetationsmanagement}

Wesentlich war die Entwicklung eines räumlich differenzierten Vegetationsmanagementkonzepts, welches drei Strecken unterscheidet (Hofbauer et al., 2006; Habersack et al., 2008a):

- Sensible Strecken in Siedlungsgebieten (Vegetationsmanagement und Pflege erforderlich).

- Übergangsstrecken jeweils vor Siedlungsgebieten sowie z. B. Brücken (eingeschränktes Vegetationsmanagement und Pflege erforderlich).

- Vegetationsdynamische Strecken im Freiland (keine Pflege notwendig).

Generell kann gesagt werden, dass im Siedlungsgebiet durch die Sensibilität der Hochwasserspiegellagen der Spielraum für die Vegetation begrenzt ist, während im Freiland mit Vegetation ein Vorteil für die fließende Retention besteht (erhöhte Rauigkeiten und Retentionswirkung; Habersack et al., 2008b). Die Ergebnisse aus FloodRisk II zeigen, dass der Einsatz (ohne Berücksichtigung hydraulischer und morphologischer Aspekte der Flüsse) des räumlich differenzierten Vegetationsma- nagements auf die Flüsse Österreichs grundsätzlich gegeben ist („starres System“). Der Einsatz hydrodynamischer Modellierungen zeigte, dass eine individuelle Betrachtung der Flüsse (z. B. Flusstyp, Gefälle, ...) bzw. von Teilbereichen (z. B. Brücken) unbedingt erforderlich ist, um eine optimale, flussangepasste Länge der Übergangsstrecken zu erhalten („flexibles System ${ }^{\prime}=\mathrm{VeMa}_{\mathrm{FLOOD}}$, siehe Abb. 8).

Mit Hilfe des räumlich differenzierten Vegetationsmanagements können ganze Flussgebiete betrachtet und damit ein einheitliches Management sowie die Akzeptanz in der Bevölkerung erreicht werden. Als nächster Schritt sollte das räumlich differenzierte Vegetationsmanagement an ausgewählten Pilotgewässern umgesetzt werden.

\section{Schlussfolgerungen}

In diesem Artikel wurden drei Methoden vorgestellt, die in FloodRisk II entwickelt oder optimiert wurden. Der Schutz und die Wiederherstellung von Überflutungsflächen stellt eine der größten $\mathrm{He}$ rausforderungen des integrierten Hochwassermanagements dar (v. a. in Bezug auf die Raumplanung). Gleichzeitig ist ein enormer Nutzungsdruck gegeben. Mit der Floodplain Evaluation Matrix FEM existiert nun eine Methode, wie Überflutungsflächen objektiv auf Einzugsgebietsebene und/oder Streckenebene evaluiert werden können. Wichtig wäre es, auch die gesetzlichen Voraussetzungen im Wasserrechtsgesetz aber auch in den Raumordnungsgesetzen zu schaffen, um den bisher aufgetretenen Summationseffekt (sukzessiver Wegfall der Überflutungsflächen mit Problem der Einzelbewilligung) einzudämmen und wo möglich ehemalige Überflutungsflächen durch Dammabrückungen etc. wiederzugewinnen.

Der minimale flussmorphologische Raumbedarf trägt den bei großen Hochwässern auftretenden morphologischen Veränderungen (Erhöhung der Flussbreite, Laufverlagerungen etc.) Rechnung. Es sollte mindestens die drei- bis siebenfache Breite (gemessen ab der Böschungsoberkante), das ist die ein- bis dreifache Breite links und rechts des Flusses von Verbauung und anderer höherwertiger Nutzung freigehalten werden. Wo möglich ist der maximale flussmorphologische Raumbedarf (potenzielles Fluss-Auensystem) anzustreben. Das räumlich differenzierte Vegetationsmanagement $\left(\mathrm{VeMa}_{\text {Flood }}\right)$ dient der Verbesserung der Retentionswirkung von ÜberflutungsflächenimFreiland beigleichzeitiger Sicherstellung von nicht zu hohen Wasserspiegellagen im Siedlungsgebiet.

\section{Danksagung}

Die AutorInnen bedanken sich beim Lebensministerium und BMVIT sowie der EU für die Beauftragungen der jeweiligen Projekte.

\section{Korrespondenz:}

Ao. Univ.Prof. DI Dr. Helmut Habersack, DI Bernhard Schober, DI Gerald Krapesch, DI Elisabeth Jäger, DI Dr. Christoph Hauer Institut für Wasserwirtschaft, Hydrologie und Konstruktiven Wasserbau (IWHW), Department für Wasser-AtmosphäreUmwelt (WAU)

Universität für Bodenkultur Wien

Muthgasse 107, A-1190 Wien

E-Mail: helmut.habersack@boku.ac.at;

Ao. Univ. Prof. DI Dr.Susanne Muhar, DI Sabine Preis, Mag. Michaela Poppe, DI Michael Weiss,

Institut für Hydrobiologie und Gewässermanagement $(\mathrm{HG})$ Department für Wasser-Atmosphäre-Umwelt (WAU) Universität für Bodenkultur Wien Max Emanuel-Straße 17

A-1180 Wien

E-Mail: susanne.muhar@boku.ac.at 